\title{
The Pathology of Lymphocytes, Histiocytes, and Immune Mechanisms in Mycobacterium tuberculosis Granulomas
}

\author{
Reubina Wadee ${ }^{1 \star}$ and Ahmed A. Wadee ${ }^{2}$ \\ ${ }^{1}$ Department of Anatomical Pathology, School of Pathology, Faculty of Health Sciences, University of the Witwatersrand, and National Health \\ Laboratory Service (NHLS), Johannesburg, South Africa; ${ }^{2}$ South African Technology Network, Durban, South Africa
}

\begin{abstract}
Granuloma formation is the pathologic hallmark of tuberculosis (TB). Few studies have detailed the exact production of cytokines in human granulomatous inflammation and little is known about accessory molecule expressions in tuberculous granulomas. We aimed to identify some of the components of the immune response in granulomas in HIVpositive and -negative lymph nodes. We investigated the immunohistochemical profiles of CD4 ${ }^{+}, \mathrm{CD}^{+}, \mathrm{CD}^{+} 8^{+}, \mathrm{Th}^{-17}$, Forkhead box P3 (FOXP3) cells, accessory molecule expression (human leukocyte antigen [HLA] classes I and II), and selected cytokines (interleukins 2, 4, and 6 and interferon- $\gamma$ ) of various cells, in granulomas within lymph nodes from 10 HIV-negative (-) and 10 HIV-positive (+) cases. CD4 ${ }^{+}$lymphocyte numbers were retained in HIV- granulomas, whereas CD4+:CD8 + cell were reversed in HIV+ TB granulomas. CD68 stained all histiocytes. Granulomas from the HIV+ group demonstrated a significant increase in FOXP3 cells. Interleukin-2 cytoplasmic expression was similar in both groups. Interferon-gamma (IFN- $\gamma$ ) expression was moderately increased, IL-6 was statistically increased and IL-4 expression was marginally lower in cells from HIV- than HIV+ TB granulomas. Greater numbers of cells expressed IFN- $y$ and IL-6 than IL-2 and IL-4 in HIV- TB granulomas. This study highlights the varied cytokine production in HIV-positive and -negative TB granulomas and indicates the need to identify localized tissue factors that play a role in mounting an adequate immune response required to halt infection. Although TB mono-infection causes variation in cell marker expression and cytokines in granulomas, alterations in TB and HIV coinfection are greater, pointing toward evolution of microorganism synergism.
\end{abstract}

\section{INTRODUCTION}

Mycobacterium tuberculosis (Mtb) is a respiratory pathogen that gives rise to tuberculosis (TB) which persists as a worldwide health burden. ${ }^{1-3}$ Macrophages drawn to areas of bacterial deposition reside within the parenchyma, where they form granulomas, the pathological hallmark of Mtb infection. ${ }^{4,5}$ Although granulomas may contain bacilli, they paradoxically facilitate mycobacterial survival by attraction and infection of host cells by organisms at the site. ${ }^{6,7}$ Within local lymph nodes, antigen-presenting cells process and present bacterial antigens along with human leukocyte antigen (HLA) class II antigens to $\mathrm{CD} 4^{+} \mathrm{T}$ lymphocytes and to $\mathrm{CD} 8^{+}$cells via the expression of soluble antigens, together with HLA class I antigens. ${ }^{7}$

T-helper cells have been divided into two groups based on their cytokine production, Th1 and Th2 cells as subsets of $\mathrm{CD}^{+}$lymphocytes. Th1 cells produce interferon-gamma (IFN-y), interleukin (IL)-2, and other pro-inflammatory cytokines. The anti-inflammatory cytokine, IL-4, produced by Th2 cells, suppresses Th1 cytokine production. ${ }^{8}$ Interleukin-6, however, has both pro- and anti-inflammatory properties and expresses either property depending on the target population., ${ }^{8,9}$

Through a series of evasive mechanisms. M. tuberculosis has shown to effectively overcome destruction by host defenses and adversely affect accessory molecule function and T-cell and host cytokine responses. ${ }^{10}$ Forkhead box P3 (FOXP3) T-regulatory cells (Tregs), characterized by a CD4 ${ }^{+}$ CD25 $5^{+}$FOXP3 immunophenotype, ${ }^{10-12}$ have been shown to suppress $\mathrm{CD} 4^{+} \mathrm{T}$-helper cell function, preventing the host's

\footnotetext{
*Address correspondence to Reubina Wadee, University of the Witwatersrand/National Health Laboratory Service (NHLS), Rm. 3L30, University of the Witwatersrand, School of Pathology, 7 York Rd., Parktown, Johannesburg 2193, Republic of South Africa. E-mail: reubinawadee@gmail.com
}

adaptive immune response to Mtb. ${ }^{10-13}$ TB has been associated with raised levels of FOXP3, which further inhibits protective T-cell responses. It has been suggested that Tregs maintain the balance of an antimicrobial response and prevent excess host tissue destruction. ${ }^{11} \mathrm{~A}$ Th-cell subgroup producing IL-17, or Th17 cells, may act as a first line of defense by initiating inflammation. ${ }^{14}$

HIV increases the risk of acquiring TB throughout the course of HIV infection with an increased likelihood of disease progression. ${ }^{15-18}$ In patients with high CD4 counts, classic, well-formed granulomas were observed, whereas patients with low CD4 counts in whom HIV has progressed have illdefined, vague granuloma formation in which there is extensive necrosis and a paucity of Langhans giant cells and epithelioid histiocytes. ${ }^{15}$ Infection by Mtb may occur early in HIV infection because of the presence of dysfunctional HIVinfected cells, such as T lymphocytes and histiocytes which are incorporated into granulomas possibly contributing to the formation of poorly formed or disrupted granulomas. ${ }^{15,19}$ Whereas HIV infection of macrophages encourages Mtb growth, dual infection decreases the viability of macrophages and prevents apoptosis of Mtb-infected macrophages. ${ }^{14,15,20}$

HIV has been shown to destroy T cells with rapid turn-over and to alter cytokine expression in Mtb granulomas, with IL-2 production by Mtb-specific T cells increasing susceptibility to infection by HIV. ${ }^{5,19}$ The continuous antigenic mediated immune stimulation eventually exhausts the host's capabilities to contain either TB or HIV with subsequent uncontrolled disease. This adds to the suggestion of a dual susceptibility model.

There have been only a few studies detailing the exact production of cytokines in human granulomatous inflammation and little is known of the expression of accessory molecules in tuberculous granulomas. The present study therefore aimed at identifying some of the components of the immune response in human tissue granulomas in both HIV-positive and -negative lymph nodes. In addition, the study assessed 
localized cell-mediated effects as would be seen in $M$. tuberculosis granulomas.

\section{MATERIALS AND METHODS}

Archived tissue blocks of surgically excised lymph nodes that were previously diagnosed as granulomatous inflammation with positive Ziehl-Neelsen stain were retrieved from the Department of Anatomical Pathology at our institution following ethical clearance. Ten lymph nodes $(n=10)$ from HIV-negative patients and $10(n=10)$ from HIV-positive patients containing TB granulomas were used. The lymph nodes were derived from peripheral sites, and none were derived from the thoracic cavity. The tissue sections were examined, and the original diagnoses confirmed. Well-formed granulomas were documented in all cases from HIV-negative patients. The lymph nodes from patients with low CD4 counts were not well formed and demonstrated extensive necrosis and an abundance of neutrophils. The HIV-positive patients were not on antiretroviral therapy at the time of biopsy. As such, only a small number of cases were identified. The patients had CD4 counts varying from 30 cells $/ \mu \mathrm{L}$ to 735 cells $/ \mu \mathrm{L}$.

All lymph nodes were stained with Periodic Acid-Schiff, thereby excluding fungal infections and nontuberculous mycobacteria. The diagnosis of TB was based on clinical, radiological, and histological findings that were suggestive of TB but without confirmation by culture or molecular studies. Paraffin-embedded tissue blocks had originally been fixed in $10 \%$ neutral buffered formalin for a period of 12-48 hours before tissue sectioning and staining according to the established departmental standard operating procedure.

Immunohistochemistry. Consecutive tissue sections were cut at $4 \mu \mathrm{m}$, from paraffin blocks, and were floated onto slides, and dried overnight at $60^{\circ} \mathrm{C}$. CD4, CD8, and CD68 immunohistochemical stains were optimized according to the standard operating procedure within our department. For the remainder of antibodies used in this study, the concentration for each stain was deemed optimal when lymphocytes and histiocytes showed crisp cytoplasmic and membranous staining with minimal background staining, according to the manufacturers' instructions. A similar approach was used for FOXP3 until desired intranuclear staining was observed. Immunohistochemical staining of tissue sections used the avidin-biotin method to apply primary antibody. These included CD 4 (BioGenex, Fremont, CA, Clone 1F6, Neat), CD 8 (Leica Microsystems, United Kingdom, Clone 4B11, I:100), CD 68 (DAKO, Glostrup, Denmark, Clone PG-M1, 1:100), IL-17 (R\&D Systems, United Kingdom, 1:5), HLA class I (Santa Cruz Biotechnology, Inc., Santa Cruz, CA, Clone B-D11, 1:10), HLA class II (Santa Cruz Biotechnology, Inc., Clone TAL 1B5, I: 300), IL-2 (Santa Cruz Biotechnology, Inc., Clone N7.48A, 1: 50), IFN-y (Santa Cruz Biotechnology, Inc., Clone H-145, 1: 75), IL-4 (Abcam, Cambridge, United Kingdom, Clone 1:50), IL-6 (Santa Cruz Biotechnology, Inc., Clone 1, 1:400), and FOXP3 (R\&D Systems, Abingdon, United Kingdom, 1:50). Thereafter, the sections were washed with Tris-buffered saline (pH 7.6). Immunohistochemistry was performed with an automated staining machine (DAKO Autostainer Link 48, Denmark), using the DAKO EnVision FLEX Target Retrieval Solution (DAKO, Glostrup, Denmark), which has high $\mathrm{pH}$ for antigen retrieval. 3,3' diaminobenzidine hydrochloride solution (DAB, Sigma-Aldrich/Merck, Darmstadt, Germany) was used as the chromogen (brown). Tissue sections were counterstained with Meyer's hematoxylin.
Negative control tissue sections used peroxidase-labeled secondary anti-sera without the primary antibody. Positive tissue controls, drawn from the departmental stock of reactive lymph nodes, were used for all immunohistochemical stains.

A limitation of the study is the small number of cases included. In addition, this study did not use multiplex immunofluorescence or in situ hybridization techniques as these techniques are not available in our laboratory. The use of immunohistochemical stains on sequential tissue sections allows for the possibility of some cells being cut away and not being present on sequential tissue sections.

Immunohistochemical assessment. Weak, pale to intense dark membrane and cytoplasmic staining of lymphocytes and histiocytes was considered positive for all immunohistochemical stains, except FOXP3. Forkhead box P3 was deemed positive if any degree of nuclear staining was identified. Positive staining for all markers was assessed in the relevant cells contained within and in areas surrounding granulomas.

Representative areas surrounding granulomas were identified by scanning on a low-power $\times 4$ objective. Within these areas, the number of positive cells stained with each of the immunohistochemical stains was counted, using an eyepiece graticule containing 100 squares fitted to an Olympus BX41 (Olympus Corporation, Tokyo, Japan) microscope at $\times 10$ ocular and $\times 40$ objective. Five of the most representative highpower fields were selected from each tissue section, and the same areas were compared in each lymph node such as the interface between necrotic foci and granulomatous inflammation as well as peripheries of granulomatous inflammation and foci adjacent to granulomas. This ensured that similar regions were assessed for each of the different stains. In each field using the $\times 40$ objective, five areas with the most intense staining were identified. In each of these areas, groups of 10 lymphocytes and histiocytes were counted and the number of positive staining cells from each group of 10 was recorded. Thus, each field provided a total of 50 cells. As five such fields were examined per slide, this provided evaluation of a total of 250 cells. The grid ensured that foci counted in each area were not duplicated. The same areas were compared in granulomas from both HIV-positive and -negative lymph nodes, comparing areas at the interface between the granulomatous inflammation and the necrotic zone with areas at the periphery of the granulomatous infiltrate and areas within the lymph node immediately adjacent to the granuloma.

The number of positive cells out of a total of 250 was recorded and averages obtained. Evaluated fields were marked on the slides, and the area matched on each slide to ensure the same area was examined for each stain.

Ethics. The study protocol was approved by our Institutional Human Research Ethics Committee; clearance number (M091020).

Statistical analysis. Data obtained from various observations were entered into a Microsoft Excel 2007 program (Microsoft Corporation, Redmond, WA). Statistical parameters of positivity for all stains were obtained for each set of observations. Statistical significance was ascertained using Student's two-tailed $t$-test (unpaired $t$-test).

\section{RESULTS}

Figures $1 \mathrm{~A}$ and $\mathrm{B}$ demonstrate TB granulomas in lymph nodes from HIV-negative and -positive lymph nodes, 


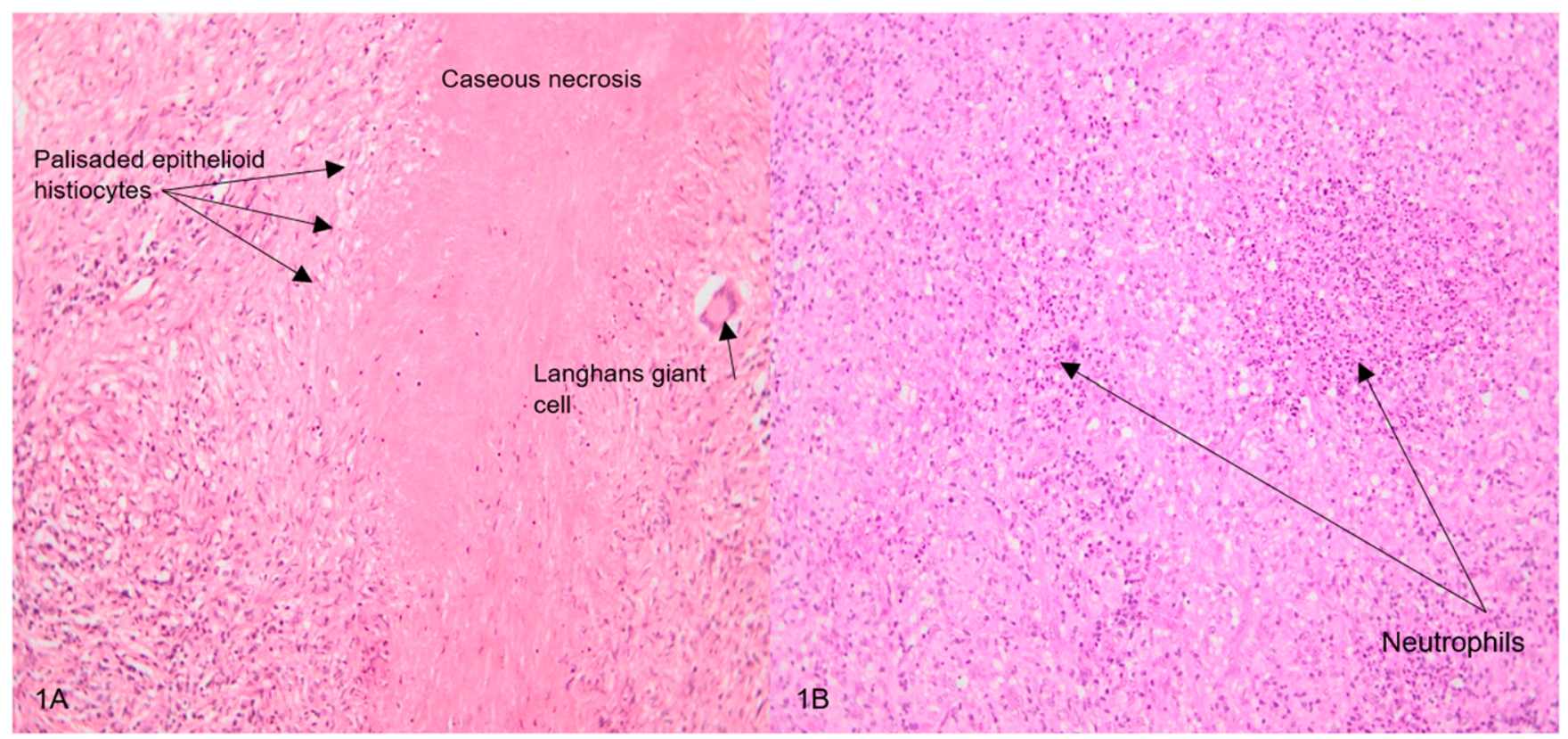

FIGURE 1. (A) Histological features of a lymph node showing classic granulomatous inflammation with caseous necrosis, palisaded epithelioid histiocytes (three arrows), and a Langhans giant cell in an HIV-negative case. (B) TB granulomas in a lymph node from an HIV-positive case showing poorly formed granulomatous inflammation with an abundance of neutrophils (two long thin arrows). (H\&E, $2 \mu \mathrm{m}$ section; original magnification: $\times 200)$. This figure appears in color at www.ajtmh.org.

respectively. HIV-negative TB granulomas showed approximately 1.5 times more CD4 ${ }^{+}$cells than those in HIV-positive granulomas $(P<0.0001)$, (Figure 2 , denoted by * and Figure 3 ).

$\mathrm{CD}^{+}$cells in granulomas from HIV-negative lymph nodes were identified at peripheries of granulomas forming a peripheral cuff of cells, in contrast to $\mathrm{CD}^{+}$cells which were scattered throughout TB granulomas in HIV-positive lymph nodes, without specific localization (Figure 4). Although our study found that the percentage of $\mathrm{CD}^{+}$and $\mathrm{CD}^{+}$staining cell numbers were similar in the HIV negative TB granulomas, there was a significant decrease in the numbers of $\mathrm{CD}^{+}$cells compared with $\mathrm{CD}^{+}$cells $(P<0.0001$, denoted by \#) in HIVpositive TB granulomas (Figure 2 ratio of 1:1.66). The percentages of IL-17 cells were similar, and all histiocytes in granulomas stained positively with CD68 (Figure 2). Higher

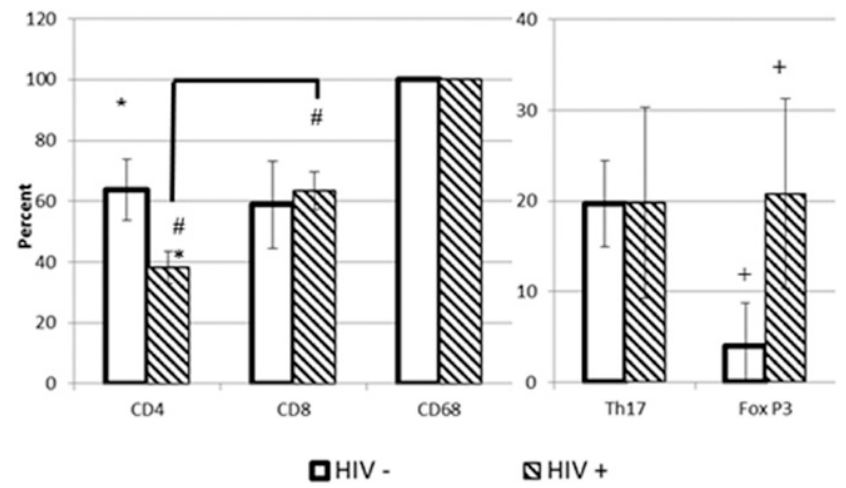

Figure 2. Percentage of CD4, CD8, CD68, Th17, and FOXP3 in corresponding areas from HIV- and HIV+ TB granulomas (see text for details). ${ }^{\star} P<0.0001$ : CD4 HIV- vs. HIV+ patients \# $P<0.0001$ : CD4 compared with CD8 in HIV + patients; ratio of CD4:CD8 is 1:1.66. $+P<$ 0.001: FOXP3 HIV- vs. HIV+ patients. FOXP3 = Forkhead box P3. numbers of nuclear FOXP3 staining were noted in TB granulomas from HIV-positive cases than those identified in HIV negative TB granulomas $(P<0.001$ denoted by + , Figures 2 and 5).

Assessments of HLA class I and HLA class II expression on cells in TB granulomas from the two groups showed a moderate but insignificant increase of 6\% HLA class I expression on cells in HIV-positive TB granulomas. All cells expressed HLA class II antigens in TB granulomas, regardless of HIV status.

Identification of pro- and anti-inflammatory cytokines in granulomas. Pro-inflammatory cytokines: IL-2 and IFN- $\gamma$. Interleukin-2 expression was similar in the two groups (Figure 6). Cells in HIV-negative TB granulomas showed a moderate $10 \%$ greater staining of IFN- $\gamma$ than cells in HIVpositive TB granulomas. Comparison of IL-2 and IFN-Y on cells in HIV-negative TB granulomas showed a significant 2 -fold increase in IFN- $\gamma$ staining $(P<0.05$, denoted by *). However, there was a very slight increase in cells staining with IFN-y compared with IL-2 in HIV-positive granulomas.

Anti-inflammatory cytokines: IL-4 and IL-6. Interleukin-4 cell staining was $13 \%$ greater in TB granulomas from HIV-positive lymph nodes than cells in HIV-negative granulomas. Based on previous studies, IL- 6 was placed in the group of the antiinflammatory cytokines. ${ }^{8,9}$ Interleukin- 6 cellular expression in HIV-negative TB granulomas was significantly increased compared with that observed in HIV-positive granulomas $(P<$ 0.0001 , denoted by !!; Figure 6). Comparisons of IL-4 versus IL-6 staining within granulomas of each group revealed that a significantly higher proportion of cells stained with IL-6 than IL-4 in HIV-negative TB granulomas $(P<0.05$, denoted by \#; IL-4:IL-6 is 1:1.30). Conversely, significantly more cells stained with IL-4 than IL-6 in HIV-positive TB granulomas $(P<$ 0.0001 , denoted by +, Figure 6); ratio of IL-4 to IL-6 is 2.64:1. In summary, TB granulomas from HIV-positive lymph nodes 


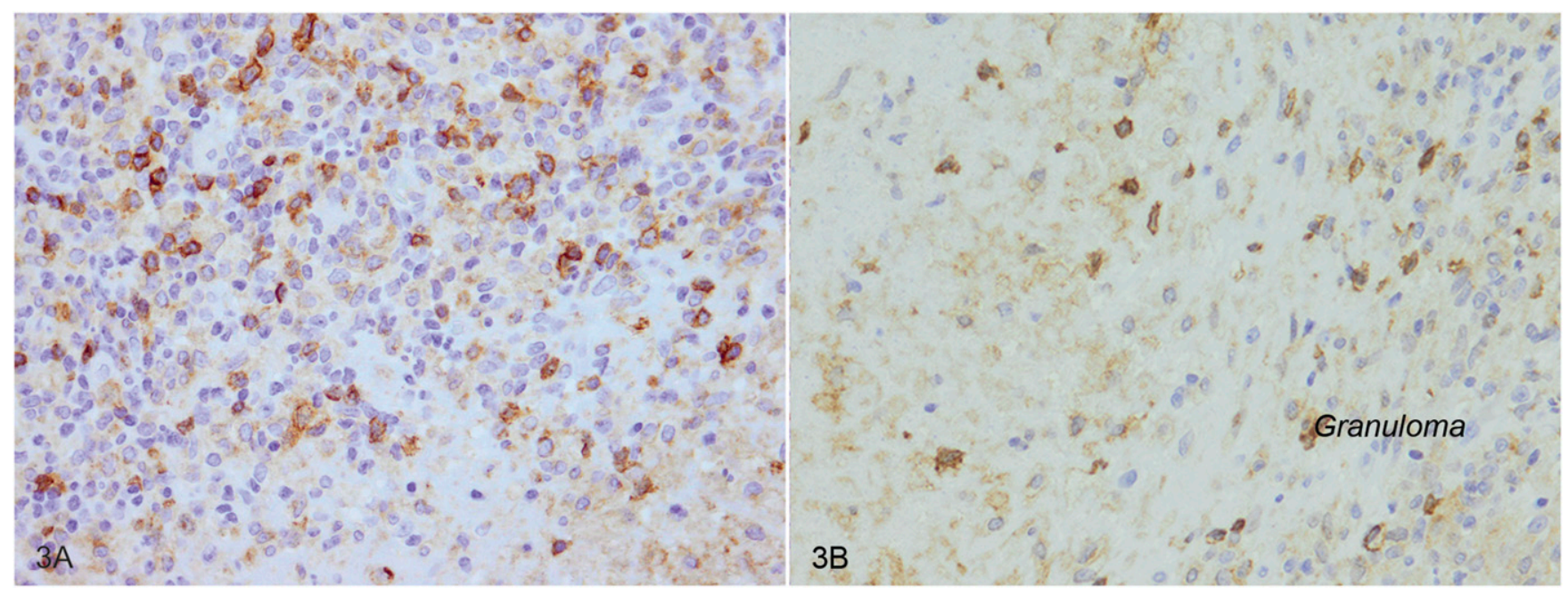

FIGURE 3. (A) $\mathrm{CD}^{+}$cells in a TB granuloma from an HIV-negative case (B) CD4 staining in a TB granuloma from an HIV-positive case corresponding to a similar area from a lymph node from an HIV-negative case. (CD4, $4 \mu \mathrm{m}$ section; original magnification: $\times 400$.) This figure appears in color at www.ajtmh.org.

exhibited significantly greater amounts of IL-4 than IL-6 $(P<$ 0.0001 denoted by + , Figure 6), whereas the expression of IL-6 was far greater than IL-4 in TB granulomas from HIV-negative nodes $(P<0.05$ denoted by \#, Figure 6$)$.

TB granulomas from the HIV-negative lymph nodes showed both a pro-inflammatory and an anti-inflammatory cytokine profile. High percentages of positive staining with IFN-y antibodies in this group relative to TB granulomas in the HIV-positive group were noted. TB granulomas from the HIV-negative group showed a marginally lower percentage of IL-2 positivity than that seen in TB granulomas from the HIV-positive group. In addition, the TB granulomas in the HIV-negative group showed marked elevation in the percentage of IL6-positive cells in comparison to the TB granulomas from HIV-positive patients. The percentage of IL-4-positive cells was approximately $13 \%$ lower in TB granulomas from HIVnegative nodes than TB granulomas from HIV-positive nodes. The percentage of IL-6-positive cells was, however, much higher than those of IL-4 in TB granulomas from the HIVnegative group (Figure 7).

In the TB granulomas from the HIV-positive group, the cytokine profile showed high expression of the anti-inflammatory marker IL-4. In this group, the percentage of IL-4 positive cells far exceeded that of IL-6.

\section{DISCUSSION}

Locally infected tissue requires a finely tuned inflammatory and anti-inflammatory response in an attempt to halt infection. ${ }^{3}$ Granuloma formation is the pathological hallmark of Mtb infection. ${ }^{4,5,7,19}$ TB granulomas in the HIV-negative lymph nodes in the present study show preservation of $\mathrm{CD}^{+}$cell counts as well as a CD4+: CD8 ${ }^{+}$ratio of approximately $1: 1$ with high numbers of $\mathrm{CD}^{+}$cells at the peripheries. Decreased numbers of CD4 ${ }^{+} \mathrm{T}$ lymphocytes with significantly increased $\mathrm{CD}^{+}$cells in TB granulomas of HIV-positive nodes were

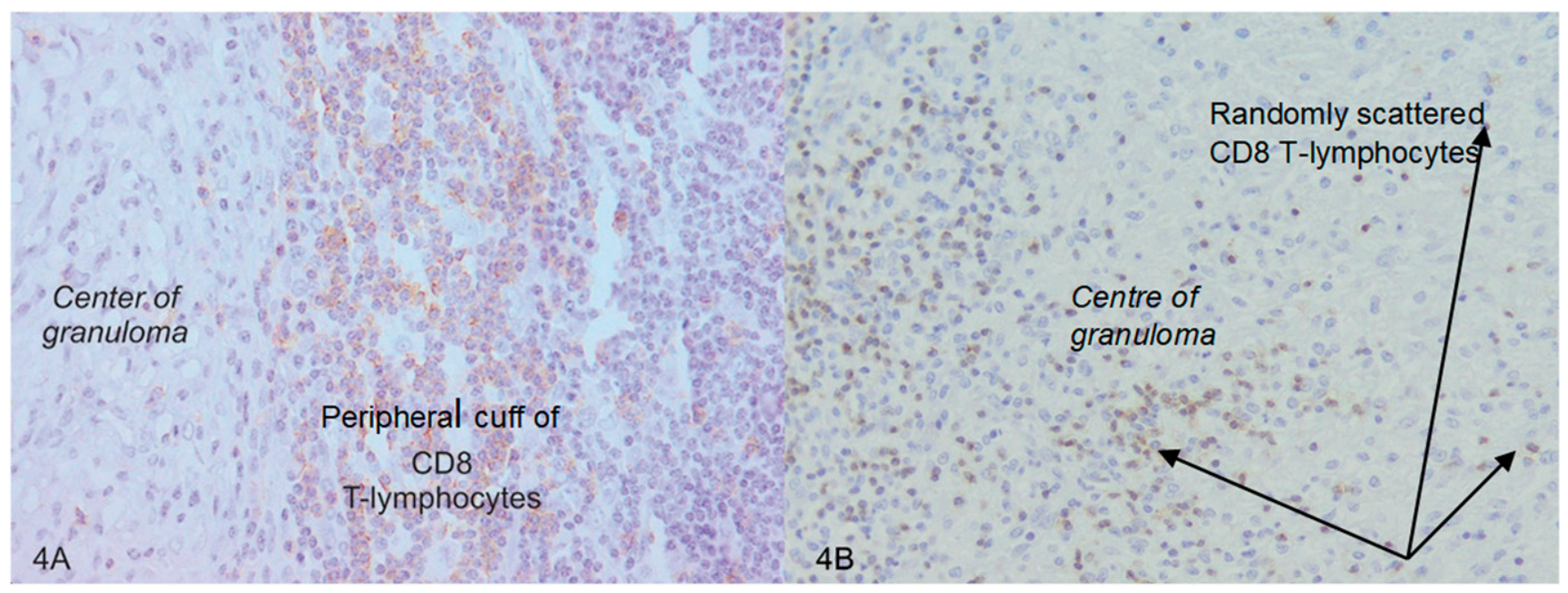

FIGURE 4. (A) $\mathrm{CD}^{+}$cells in a TB granuloma from an HIV-negative case highlighting their peripheral localization. (B) $\mathrm{CD}^{+}$cells are peripherally and centrally identified in a granuloma from an HIV-positive case (CD8, $4 \mu \mathrm{m}$ section; original magnification: $\times 200$.) This figure appears in color at www.ajtmh.org. 


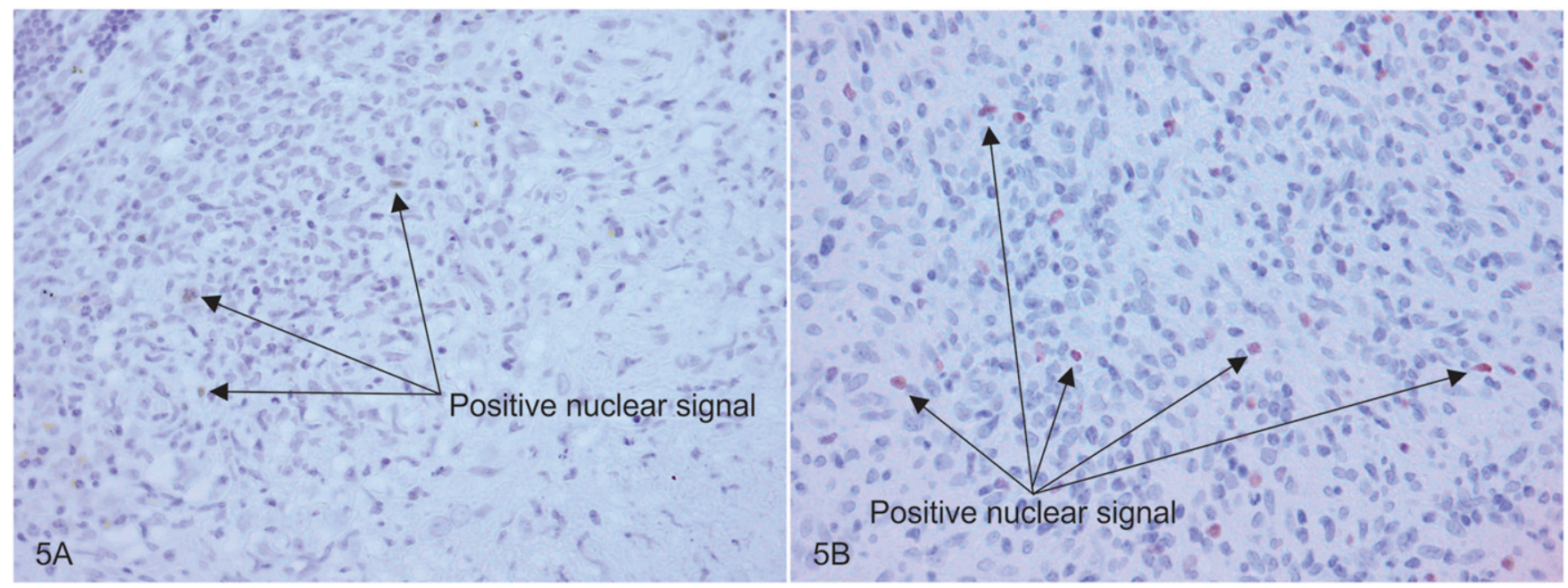

FIGURE 5. (A) FOXP3 nuclear positivity is highlighted in very few cells in a TB granuloma from an HIV-negative case. (B) Numerous FOXP3 nuclei are demonstrated in a TB granuloma from an HIV-positive case. (FOXP3, $4 \mu \mathrm{m}$ section; original magnification $\times 400$.) FOXP3 $=$ Forkhead box P3. This figure appears in color at www.ajtmh.org.

observed in our study similar to changes seen by others, in various anatomical sites and models., $21-24$ Lymph nodes from HIV-positive patients with higher CD4 counts showed better formed granuloma formation in contrast to lymph nodes from patients with lower CD4 counts. These findings are all in keeping with current knowledge of $\mathrm{CD}^{+}$destruction and depletion in HIV. ${ }^{5,6,11,15,19,22,25}$ In the present study, the HIV positive TB granulomas were found to have many $\mathrm{CD}^{+}$cells dispersed throughout granulomas and admixed with palisading epithelioid histiocytes, as opposed to forming a peripheral cuff of $\mathrm{CD}^{+}$cells in HIV-negative TB granulomas. This finding suggests that retention of $\mathrm{CD} 4^{+}$cells may be central to organization of granulomas.

Positive staining of CD68 was identified on all histiocytes in TB granulomas from both HIV-positive and -negative patients. These findings are similar to those found by others illustrating that all histiocytes within tissue granulomas in TB patients were immunoreactive with a CD68 stain. $^{26}$ The IL-17 cell counts in the granulomas of both HIV-negative and -positive TB groups were found to be similar in the present study.

The present study shows markedly elevated levels $(>5$ times) of FOXP3 in the HIV-positive TB granulomas in contrast

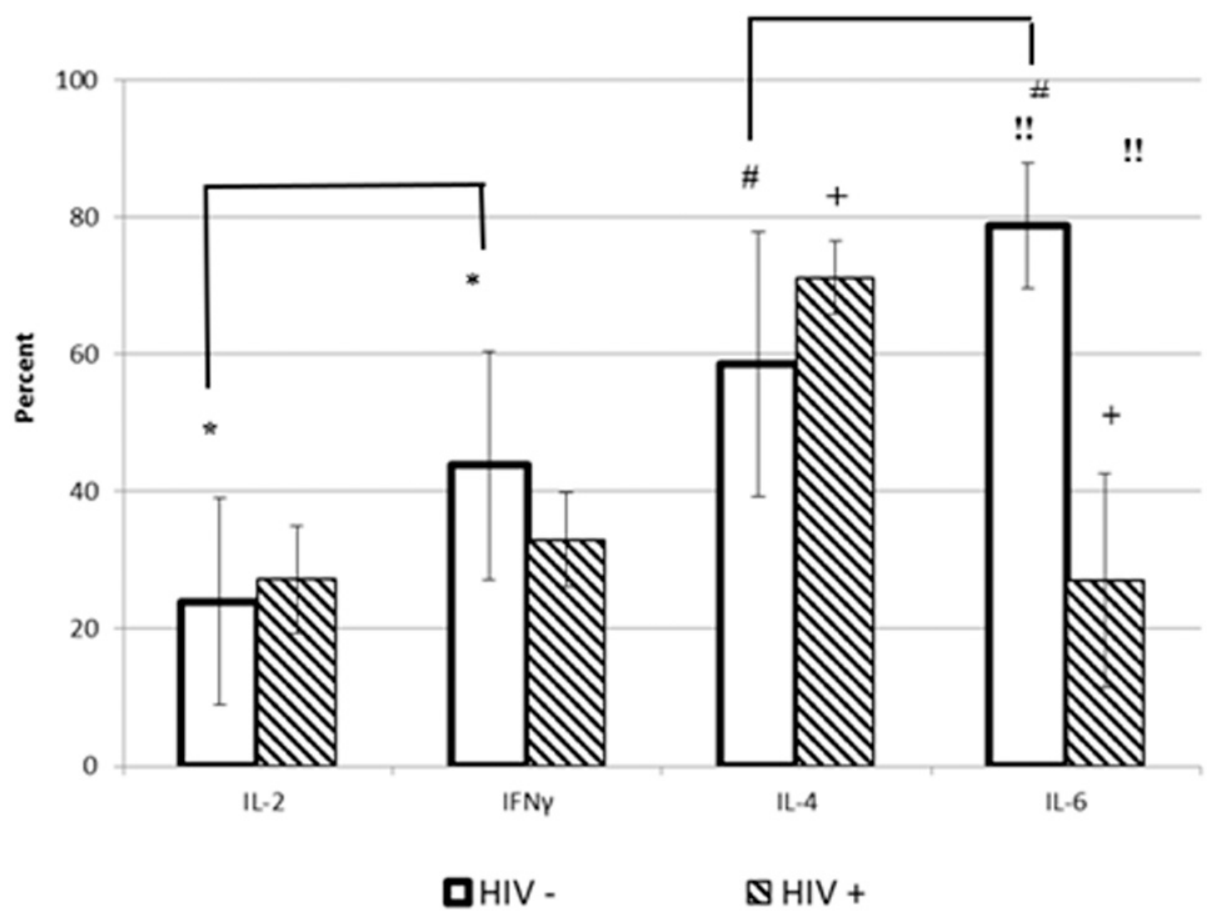

FIGURE 6. TB granuloma cellular cytokine expression. Significantly less cells expressed IL-6 in HIV+ than HIV-TB granulomas (!!P $<0.0001)$. HIVgranulomas demonstrated significantly less IL-2 than interferon-gamma (IFN- - ) $\left.{ }^{\star} P<0.05\right)$ but more IL-6 than IL-4 (\# $\left.P<0.05\right)$. HIV+ granulomas showed significantly higher IL-4 (+) than IL-6 (+); $(P<0.001)$. IL = Interleukin. 


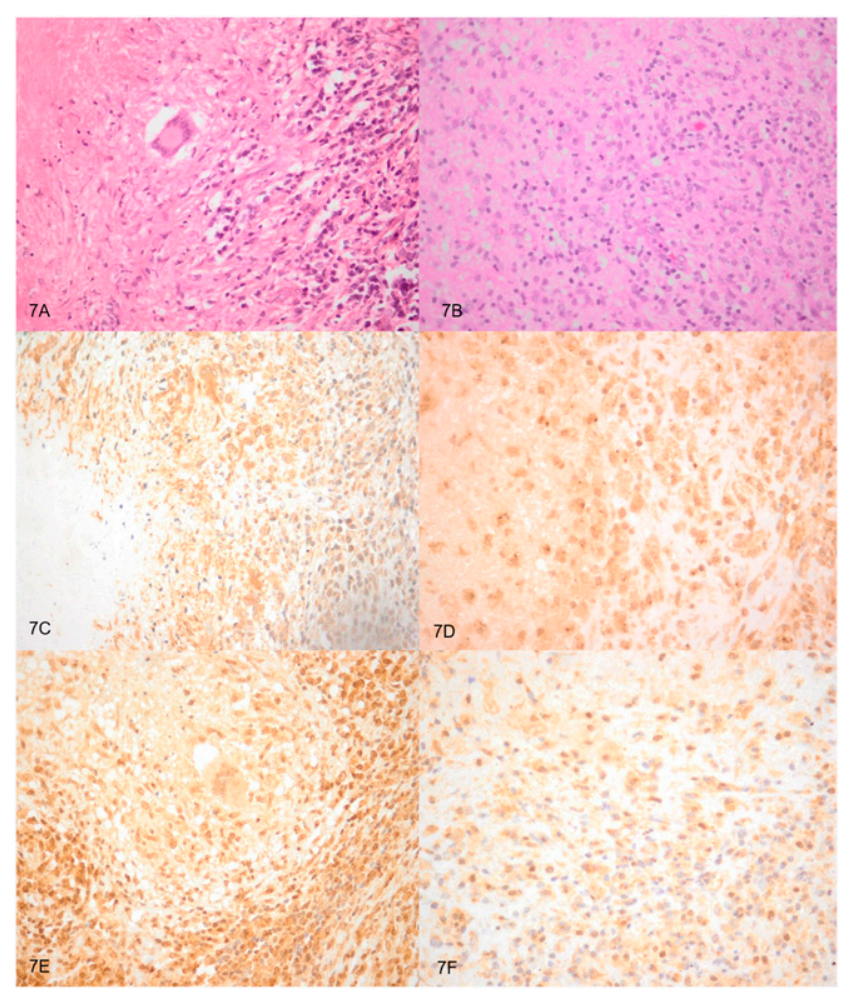

FIGURE 7. A composite image of anti-inflammatory and pro-inflammatory cytokines in HIV-negative and -positive TB granulomas. (A) TB granuloma from an HIV-negative case. (B) TB granuloma from an HIV-positive case. (C) interferon-gamma (IFN- $\gamma$ ) staining in an HIVnegative TB granuloma. (D) IL-2 staining in an HIV-positive TB granuloma. (E) IL-6 staining in an HIV-negative TB granuloma. (F) IL-4 staining in an HIV-positive TB granuloma. (A-F original magnification $\times 400)$. IL = Interleukin. This figure appears in color at www.ajtmh.org.

to the HIV-negative group, suggesting that these cells prevent extensive localized tissue destruction. Although transforming growth factor- $\beta$ (TGF- $\beta$ ) has not been included in this study, it has been documented that TGF- $\beta$ may have both proinflammatory and anti-inflammatory effects, and functions in the regulation of immune responses during infection and disease. It has been shown that TGF- $\beta$ may induce FOXP3 expression and also play a role in the suppression of immune responses via Tregs. ${ }^{27}$ Granulomas in the HIV-positive lymph nodes were mostly well formed, despite having contained higher percentages of FOXP3 cells than the HIV-negative group. Although the granulomas in this group of nodes contained $\mathrm{CD}^{+}$cells at the periphery, they did, however, contain scattered $\mathrm{CD}^{+}$cells within the inner regions, admixed with epithelioid histiocytes, supporting the notion that granuloma integrity is maintained by $\mathrm{CD} 4^{+}$cells.

Although our study indicates only marginally lower levels of IL-2 in HIV-negative TB granulomas than in HIV-positive lymph node granulomas, our study insinuates that a diminution of Mtb-specific T cells, as well as a decrease in the cytokines produced by these cells, favors the survival of both HIV and Mtb. ${ }^{19}$

The raised percentage of IFN- $\gamma$ immunoreactive cells in our study in HIV-negative TB granulomas (compared with HIVpositive TB granulomas) corroborates findings by others who demonstrated an increase in this cytokine in cases of mild to moderate Mtb infection. ${ }^{22,23}$ As IFN-y is associated with necrosis, the increased expression of IFN- $\gamma$ in the HIVassociated TB granulomas may suggest more significant necrosis in this group of TB granulomas. Our study suggests that host immunity in HIV-negative TB granulomas has a far stronger Th1 response with marked pro-inflammatory response than in HIV-positive TB granulomas. This suggests that the overall immune response in HIV-negative individuals is able to contain $M$. tuberculosis more efficiently than HIVpositive individuals.

The mean cell count of IL-4 was lower in HIV negative TB granulomas when compared to HIV positive TB granulomas. Interleukin-4 detection in TB granulomas has resulted in conflicting findings with increased or decreased levels being reported, and the Th2 response of this cytokine is contentious. ${ }^{23}$ Regardless of the debate, the role of the Th2 response in TB granulomas may not be ignored.

The present study showed a highly significant difference $(P<0.0001)$ between the percentage of IL-6-positive cells in TB granulomas of the HIV-negative group compared with TB granulomas in the HIV-positive group. Interleukin- 6 has been demonstrated to have numerous functions, including a proinflammatory role, or inducing T-cell suppression with a resultant reduction in TNF- $\alpha$ or inhibiting IFN- $-{ }^{8,28}$ It has been suggested that inhibition of TNF- $\alpha$ by IL- 6 may promote the spread of disease and that inhibition of IFN- $\gamma$ by IL- 6 may be yet another mechanism that Mtb takes advantage of in its attempt at escaping host cell defences. ${ }^{8}$ Furthermore, recent use of TNF- $\alpha$ antagonists for autoimmune conditions may result in reactivation of TB. ${ }^{29}$ It is therefore possible that IFN- $\gamma$, IL-6, and TNF- $\alpha$ blocking agents could serve to regulate the immune response and dampen host immune responses within the microenvironment of the granuloma. ${ }^{23,29}$ Overall evaluation of the cytokines in the TB granulomas from HIV-negative lymph nodes shows a fairly robust pro-inflammatory response. However, the percentages of both the antiinflammatory cytokines, IL-4 and IL-6, in TB granulomas from this group far exceed the percentages of both proinflammatory cytokines, IL-2 and IFN-Y. It is therefore postulated that although the HIV-negative lymph nodes have been able to raise a cellular response to contain Mtb, the concomitant anti-inflammatory response likely served to prevent excessive tissue damage and also curtailed the host's ability to eradicate infection. The impaired Th1 response may be further decreased in progressive HIV disease because of an increase in Tregs. ${ }^{25}$ With regard to TB granulomas in the HIVpositive group in the current study, the anti-inflammatory response is greater than the pro-inflammatory response. This implies that a Th2 response prevails with eventual progression of disease. In our study, we have shown TB mono-infection is associated with low percentages of FOXP3. However, in TB and HIV coinfection, the percentages of FOXP3 were significantly increased, suggesting that FOXP3 cells may dampen the inflammatory cell response to curtail localized tissue destruction.

This study has highlighted the varied cytokine production in TB granulomas in HIV-positive and -negative lymph nodes and indicates the need to identify localized tissue factors that play a role in preventing an adequate immune response required to halt infection. Collectively, the alterations noted in TB and HIV coinfection suggest evolution of microorganism synergism. 
However, the use of multiplex immunofluorescence staining and in situ hybridization of cytokine mRNA may be useful for the diagnosis of TB, especially in cases which are paucibacillary. Multiplex immunofluorescence staining facilitates simultaneous assessment of various markers within a single tissue section. ${ }^{26}$ To gain a better understanding of the complex interplay between pro-inflammatory and anti-inflammatory processes within a granuloma, studies using additional cytokines could be undertaken in future.

Received October 20, 2020. Accepted for publication January 24, 2021.

Published online March 15, 2021.

Acknowledgment: We thank Eric Liebenberg his assistance with photomicrographs.

Financial support: This research has been funded by the University of the Witwatersrand.

Disclosure: The experimental work of this manuscript has been submitted and awarded by the University of the Witwatersrand for the degree of Master of Medicine, to R. Wadee.

Authors' addresses: Reubina Wadee, Department of Anatomical Pathology, School of Pathology, Faculty of Health Sciences, University of the Witwatersrand, and National Health Laboratory Service (NHLS), Johannesburg, South Africa, E-mail: reubinawadee@gmail.com. Ahmed A. Wadee, South African Technology Network, Durban, South Africa, E-mail: ahmed@satn.co.za.

\section{REFERENCES}

1. Flynn J, Chan J, Lin $P, 2011$. Macrophages and control of granulomatous inflammation in tuberculosis. Mucosal Immunol 4: 271-278.

2. Cardona P, Cardona PJ, 2019. Regulatory T cells in Mycobacterium tuberculosis infection. Front Immunol 10: 2139.

3. Wong EA et al., 2020. IL-10 impairs local immune response in lung granulomas and lymph nodes during early Mycobacterium tuberculosis infection. J Immunol 204: 644-659.

4. Silva Miranda M, Breiman A, Allain S, Deknuydt F, Altare F, 2012. The tuberculous granuloma: an unsuccessful host defence mechanism providing a safety shelter for the bacteria? Clin Dev Immunol 2012: 139127.

5. Pawlowski A, Jansson M, Sköld M, Rottenberg ME, Källenius G, 2012. Tuberculosis and HIV co-infection. PLos Pathog 8: e1002464.

6. Ramakrishnan L, 2012. Revisiting the role of the granuloma in tuberculosis. Nat Rev Immunol 12: 352-366.

7. Kumar V, Abbas AK, Aster JC, 2020. Robbins and Cotran Pathologic Basis of Disease, 10th edition. Philadelphia, PA: Elsevier, 367-374.

8. Scheller J, Chalaris A, Schmidt-Arras D, Rose-John S, 2011. The pro- and anti-inflammatory properties of the cytokine interleukin-6. Biochim Biophys Acta 1813: 878-888.

9. Fuster JJ, Walsh K, 2014. The good, the bad, and the ugly of interleukin-6 signaling. EMBO J 33: 1425-1427.

10. Shafiani S, Tucker-Heard G, Kariyone A, Takatsu K, Urdahl KB, 2010. Pathogen-specific regulatory $T$ cells delay the arrival of effector T cells in the lung during early tuberculosis. J Exp Med 207: 1409-1420.

11. Ottenhoff THM, 2012. The knowns and unknowns of the immunopathogenesis of tuberculosis [State of the art]. Int $J$ Tuberc Lung Dis 16: 1424-1432.

12. Suchard MS, Mayne E, Green VA, Shalekoff S, Donninger SL, Stevens WS, Gray CM, Tiemessen CT, 2010. FOXP3 expression is upregulated in CD4+T cells in progressive HIV-1 infection and is a marker of disease severity. PLoS One 5: e11762.

13. Hirsch CS, Baseke J, Kafuluma JL, Nserko M, Mayanja-Kizza H, Toossi Z, 2016. Expansion and productive HIV-1 infection of Foxp3 positive CD4 T cells at pleural sites of HIV/TB coinfection. J Clin Exp Immunol 1. Available at: http://www. opastonline.com/wp-content/uploads/2016/11/expansion-andproductive-hiv-1-infection-of-foxp3-positive-cd4-t-cells-at-pleuralsites-of-hivtb-co-infection-jcei-16-007.pdf. Epub 2016 Oct 31.

14. Mortaz E, Varahram M, Farnia P, Bahadori M, Masjedi M, 2012. New aspects in immunopathology of Mycobacterium tuberculosis. ISRN Immunol 2012: 1-11.

15. Walker NF, Meintjes G, Wilkinson RJ, 2013. HIV-1 and the immune response to TB. Future Virol 8: 57-80.

16. Kaplan R, Hermans S, Caldwell J, Jennings K, Bekker L-G, Wood $R, 2018$. HIV and TB co-infection in the ART era: CD4 count distributions and TB case fatality in Cape Town. BMC Infect Dis 18: 356.

17. Tavares AM, Fronteira I, Couto I, Machado D, Viveiros M, Abecasis AB, Dias S, 2017. HIV and tuberculosis co-infection among migrants in Europe: a systematic review on the prevalence, incidence and mortality. PLoS One 12: e0185526.

18. Ahmed A, Rakshit S, Vyakarnam A, 2016. HIV-TB co-infection: mechanisms that drive reactivation of Mycobacterium tuberculosis in HIV infection. Oral Dis 22 (Suppl 1): 53-60.

19. Diedrich CR, Flynn JL, 2011. HIV-1/Mycobacterium tuberculosis coinfection immunology: how does HIV-1 exacerbate tuberculosis? Infect Immun 79: 1407-1417.

20. Pathak S, Wentzel-Larsen T, Åsjö B, 2010. Effects of in vitro HIV-1 infection on mycobacterial growth in peripheral blood monocytederived macrophages. Infect Immun 78: 4022-4032.

21. O'Garra A, Redford PS, McNab FW, Bloom Cl, Wilkinson RJ, Berry MPR, 2013. The immune response in tuberculosis. Annu Rev Immunol 31: 475-527.

22. Geldmacher C, Zumla A, Hoelscher M, 2012. Interaction between HIV and Mycobacterium tuberculosis: HIV-1-induced CD4 T-cell depletion and the development of active tuberculosis. Curr Opin HIV AIDS 7: 268-274.

23. Abebe $F, 2012$. Is interferon-gamma the right marker for bacille Calmette-Guérin-induced immune protection? The missing link in our understanding of tuberculosis immunology. Clin Exp Immunol 169: 213-219.

24. du Bruyn E, Peton N, Esmail H, Howlett PJ, Coussens AK, Wilkinson RJ, 2018. Recent progress in understanding immune activation in the pathogenesis in HIV-tuberculosis co-infection. Curr Opin HIV AIDS 13: 455-461.

25. Brighenti S, Andersson J, 2010. Induction and regulation of CD8+ cytolytic T cells in human tuberculosis and HIV infection. Biochem Biophys Res Commun 396: 50-57.

26. Abengozar-Muela M, Esparza MV, Garcia-Ros D, Vásquez CE, Echeveste JI, Idoate MA, Lozano MD, Melero I, de Andrea CE, 2020. Diverse immune environments in human lung tuberculosis granulomas assessed by quantitative multiplexed immunofluorescence. Modern Pathol 33: 2507-2519.

27. Worthington JJ, Fenton TM, Czajkowska BI, Klementowicz JE, Travis MA, 2012. Regulation of TGF $\beta$ in the immune system: an emerging role for integrins and dendritic cells. Immunobiology 217: 1259-1265.

28. Lin PL et al., 2010. Tumor necrosis factor neutralization results in disseminated disease in acute and latent Mycobacterium tuberculosis infection with normal granuloma structure in a cynomolgus macaque model. Arthritis Rheum 62: 340-350.

29. Harris J, Keane J, 2010. How tumour necrosis factor blockers interfere with tuberculosis immunity. Clin Exp Immunol 161: $1-9$. 